\title{
A CANONICAL FORM FOR THE ISOPERIMETRIC INEQUALITY ON MANIFOLDS OF CONFORMALLY HYPERBOLIC TYPE
}

\author{
V.M. KESEL'MAN and V.A. ZORICH
}
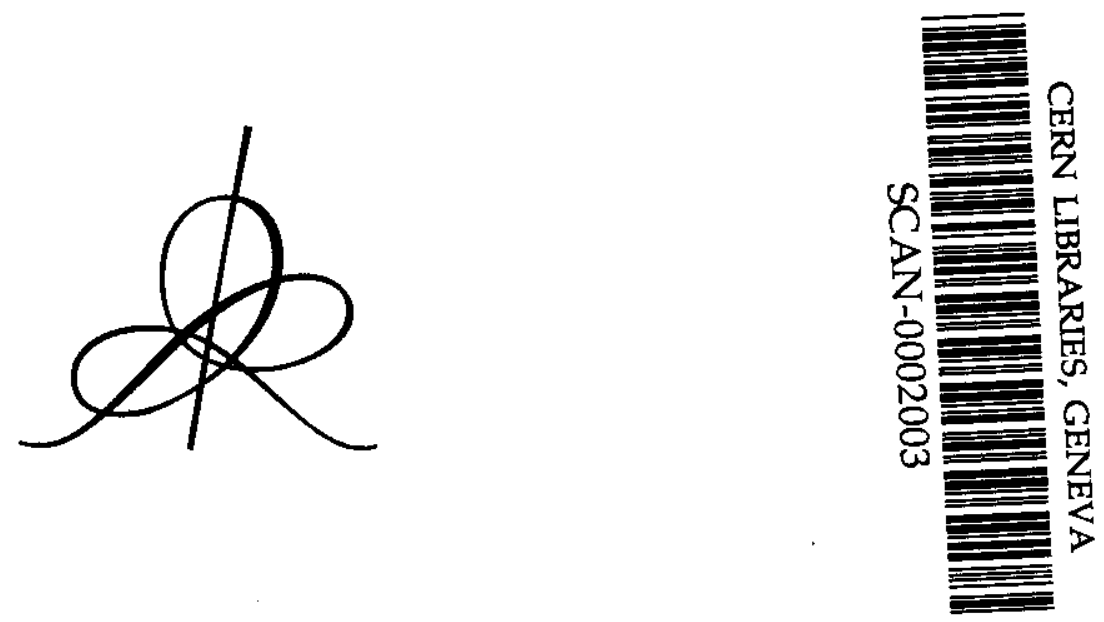

Institut des Hautes Études Scientifiques

35 , route de Chartres

91440 - Bures-sur-Yvette (France)

Avril 1999

$\mathrm{IHES} / \mathrm{M} / 99 / 23$ 


\title{
A canonical form for the isoperimetric inequality on manifolds of conformally hyperbolic type
}

\author{
V.M. Kesel'man, V.A. Zorich
}

8 April 1999

\begin{abstract}
We prove that on any Riemannian manifold of conformally hyperbolic type the maximal isoperimetric function can be reduced to the linear canonical form $P(x)=x$ by means of a conformal change of the initial Riemannian metric. In other words, the isoperimetric inequality $P(V(D)) \leq S(\partial D)$ relating the volume $V(D)$ of a domain $D$ and the area $S(\partial D)$ of its boundary can be reduced to the form $V(D) \leq S(\partial D)$ as in Lobachevski hyperbolic space.
\end{abstract}

1. Introduction. As is well known, the isoperimetric inequality is a relation of the form

$$
P(V(D)) \leq S(\partial D)
$$

relating the volume $V(D)$ of a domain $D$ and the area $S(\partial D)$ of its boundary, where $P$ is a function called the isoperimetric function of the space. Of course, only nontrivial and, as a roule, maximal isoperimetric functions are of interest. Examples are, up to a factor, the function $P(x)=x^{\frac{n-1}{n}}$ for Euclidean $n$-dimensional space $R^{n}$, and the linear function $P(x)=x$ for Lobachevski space.

Similarly to Fuclidean and Lobachevski spaces, general open Riemannian (and even sub-Riemannian) manifolds can be divided in two conformally invariant classes according to the conformal capacity of the manifold at infinity 
(see [1], [2]). If the caparity is cqual to zero (as in the case of Euclidean space), the manifold is said to be of conformally parabolic type; if the capacity is positive (as in the case of Lobachevski space), the manifold is said to be of conformally hyperbolic type.

A conformal change of metric (in physical terms a gauge transformation related to a choice of local scales) does not change the conformal type of the manifold but may change metric relations on it.

We are interested in a canonical form for the isoperimetric inequality in the conformal class of the metric of the manifold. In other words, we wish to reduce the isoperimotric inequality to a canonical form by means of a conformal change of the initial Riemannian metric.

The results we obtained in [I] allowed us to formulate the following hypothesis: on any manifold of conformally hyperbolic type the maximal isoperimetric function can be recluced to the linear form $P(x)=x$, as in Lobachevski space; and on any $n$-dimensional manifold of conformally parabolic type to the form $P(x)=x^{\frac{n}{n-1}}$, as in Euclidean space.

In the present paper we consider the first part of this hypothesis, and we answer positively the problem posed in [3].

Recall now the definition of the conformal type of a manifold. Let $G$ be a domain in an open $n$-dimensional Riemannian manifold $(M, g)$, and $C$ be a nondegenerate continum (comnected compact set which is not a point) in $G$. A pair $(C, C)$ is frequently refered to as a condenser, meaning that $G \backslash C$ is a body of the condenser, and its boundary components $\partial G$ and $\partial C$ are its plates.

The conformal capacity of the condenser $(C, G)$ is the quantity

$$
\operatorname{cap}(C, C i)=\operatorname{iuf} \int_{G}|\nabla f|^{n} d v
$$

where the infimum is taken over all smooth functions $f$ with compact support in $G$ such that $f \equiv 1$ on $C$ and $0 \leq f \leq 1$ in $G$. Obviously the conformal capacity does not change if one extends the class of functions $f$ to all functions of the Sobolev space $L_{n}^{1}(G)$ which satisfy the condition $\left.f\right|_{C}=1$ in the space $L_{n}^{1}(G)$.

A Riemannian metric $\tilde{g}$ on $M$ is conformally equivalent or conformal to the metric $g$, if $\grave{g}=\lambda^{2} g$ on $M$, where $\lambda$ is a positive regular function on $M$. 
It is easy to see, and is well known, that cap $(C, G)$ is invariant with respect to conformal changes of initial metric.

One can describe the mass of the absolute or the ideal boundary of a manifold in a conformally invariant way by means of the conformal capacity. With this purpose, consider condensers $(C, G)$ with fixed inner plate $\partial C$ and with exterior plate $\partial G$ taken to "infinity", i.e., the domain $G$ extends to the whole manifold $M$. The limit of the capacities of such condensers is cap $(C, M)$. For any given manifold $M$ the quantity cap $(C, M)$ is either positive or zero independent of the choice of a nondegenerate continuum $C$.

Thus, the vanishing or noilvanishing of the capacity depends only on the geometry of the manifold $M$ at "infinity". In exactly this sense one speaks of zero or positive conformal capacity of the manifold at infinity. Examples are Euclidean space, where cap $\left(C, R^{n}\right)=0$, and Lobachevski space, where $\operatorname{cap}\left(C, H^{n}\right)>0$.

We say that a manifold $M$ is of conformally parabolic type if the conformal capacity of the manifold at infinity is zero, i.e., $\operatorname{cap}(C, M)=0$ for any nondegenerate continuum $C \subset M$. If this capacity is positive, we say that $M$ is a manifold of conformally hyperbolic type.

This dichotomy of conformal type is obviously invariant with respect to conformal change of Riemannian metric.

2. Main results. We consider an arbitrary $n$-dimensional noncompact Riemannian manifold $M$ without boundary and an initial metric $g$. When we pass to the new metric $\tilde{g}$ conformal to $g$, the corresponding geometric objects will be denoted with a tilde.

Theorem. A noncompact Riemannian manifold $(M, g)$ is of conformally hyperbolic type if and only if there exists a metric $\tilde{g}$ conformally equivalent to $g$ such that the isoperimetric inequality

$$
\tilde{V}(D) \leq \tilde{S}(\partial D)
$$

is fulfilled in $(M, \tilde{g})$ for every regular domain $D \subset M$ with $\tilde{V}(D) \geq c$, where $c$ is a positive arbitrary small constant.

A regular domain is, as usual, a domain with piecewise smooth boundary and compact closure, so it has finite $n$-measure (volume) $\tilde{V}(D)$ and its 
boundary has finite $(n-1)$-Hausdorff measure (area) $S(\partial D)$.

Thus, the isoperimetric function of any Riemannian manifold of conformallyhyperbolic type can be reduced in the conformal class of the initial metric to $P(x)=x$ as in Lobachevski space.

Supplement 1. The inequality $(\star)$ is asymptotically sharp in the sense that $\tilde{S}(\partial D) / \tilde{V}(D)$ can be arbitrary close to one for regular domains of arbitrary large $\tilde{g}$-volume.

More precisely, for any $\varepsilon>0$ one can choose the metric $\tilde{g}$, such that $1 \leq \tilde{S}(\partial D) / \tilde{V}(D) \leq 1+\varepsilon$ for a sequence of domains $D$ that exhaust $M$.

Supplement 2. The metric $\tilde{g}$ of our Theorem can be chosen in such a way that the volume of a geodesic ball of radius $r$ with respect to this metric grows like $e^{r}$ as $r \rightarrow+\infty$.

Recall (see [4]), that the isoperimetric dimension of a manifold of infinite volume is the supremum of all numbers $m>0$, such that the function $P(x)=$ $x^{\frac{m-1}{m}}$ (defined for all $x>\varepsilon>0$ ) is an isoperimetric function of a manifold. The supremum of the isoperimetric dimensions of the manifold with respect to the metrics conformal to the initial metric of the manifold is the conformal isoperimetric dimension of the manifold (see [5]).

The Theorem formulated above then implies

Corollary. A Riemannian manifold is of conformally hyperbolic type if and only if its conformal isoperimetric dimension is $+\infty$.

3. Proofs. In this section we outline the proofs of the above statements.

Proof of Theorem. First, notice that the isoperimetric inequality $(\star)$ implies the conformal hyperbolicity of the Riemannian manifold $M$. This statement is contained in the Ahlfors-Gromov lemma [4] and in an almost explicit form for the case of a complete manifold $M$.

A similar general proof can be obtained, for example, by the following 
estimate for the conformal capacity (see [6] ur [1]):

$$
\left(\int_{v(a)}^{v(r)} \tilde{P}^{\frac{n}{1-n}}\right)^{1-n} \leq \operatorname{cap}\left(D_{a}, D_{r}\right) .
$$

Here $\left\{D_{r} \mid r \in R_{+}\right\}$are concentric compact g-geodesic balls, $v(r)=\tilde{V}\left(D_{r}\right)$, and $\tilde{P}$ is the isoperimetric function of the manifold with respect to a metric $\tilde{g}$ conformally equivalent to the Riemannian metric $g$. The metric $g$ may be supposed to be complete, since it is straightforward to realize this by a conformal change of metric.

Thus, if the isoperimetric inequality $(\star)$ holds for a metric $\tilde{g}$ conformal to the initial one, then the function $\tilde{P}$ is of the form $\tilde{P}(x)=x$ for $x>\varepsilon>0$. Therefore the integral in (1) converges as $r \rightarrow+\infty$. Thus, the manifold $M$ is of conformally hyperbolic type.

We move on to the basic statement of the theorem about the reduction of the isoperimetric function to a linear canonical form on the manifold $M$ of conformally hyperbolic type.

Take a compact set $C \subset M$ of positive capacity but with $g$-volume zero. By virtue of the conformal hyperbolicity of $M$, there exists a unique extremal function $u \in \stackrel{\circ}{L}_{n}^{1}(G), u l_{c}=1$, such that

$$
\operatorname{cap}(C, M)=\int_{M}|\nabla u|^{n} d v>0 .
$$

The function $u$, as a generalised function (distribution), satisfies in $M \backslash C$ the equation

$$
\Delta_{n} u \equiv \operatorname{div}\left(|\nabla u|^{n-2} \nabla u\right)=0,
$$

which is the Euler-Lagrange equation of this variational problem.

There are many papers devoted to properties of solutions of equation (2), called $n$-harmonic functions (e.g., see [7], [8], and the bibliography of [9]). A number of properties of classical harmonic functions (extremum principle, Harnack inequality, Harnack theorems, etc.) also hold in the general nonlinear case. In particular, it is known that $n$-harmonic functions belong to the class $C^{1, \alpha}$. Moreover, they belong to $C^{\infty}$ near points where $\nabla u \neq 0$.

By virtue of the strict extremum principle for $n$-harmonic functions, $0<$ $u<1$ everywhere in $M \backslash C$ and obviously inf $u=0$ on $M$. 
Having found $u$, we construct the new metric $g$ conformal to the Riemannian metric $g$ by letting:

$$
\tilde{g}=\left((n-1) \frac{|\nabla u|}{u}\right)^{2} \cdot g .
$$

We then check that the isoperimetric inequality $(\star)$ is fulfilled with respect to $\tilde{g}$ for arbitrary regular domain $D \subset M$.

Suppose that the compact set $C$ is a smooth hypersurface in $M$ which is diffeomorphic to an $(n-1)$-dimensional closed disk (ball). By Stokes' formula (applied to $U=D \backslash C$ ) and equation (2) we have

$$
-\int_{\partial U}|\nabla u|^{n-2} \frac{\langle\nabla u, \bar{\nu}\rangle}{u^{n-1}} d \sigma=(n-1) \int_{U} \frac{|\nabla u|^{n}}{u^{n}} d v
$$

where $\bar{\nu}$ is a unit exterior normal to $\partial U$ and $d \sigma$ is the element of $(n-1)$ Hausdorff measure on the boundary $\partial U$.

The boundary $\partial U$ of the set $U$ is the union of $C \cap \bar{D}$ and $\partial D \backslash C$, and $\langle\nabla u, \nu\rangle \geq 0$ on $C \cap \bar{D}$. Therefore, the left hand side of (4) does not decrease if one writes the integral over the set $\partial D \backslash C$ only. Moreover, the integral on the right of (4) can be extended to $D$ since $V(C)=0$. Thus, we obtain the inequality

$$
(n-1) \int_{D}\left(\frac{|\nabla u|}{u}\right)^{n} d v \leq \int_{\partial D}\left(\frac{|\nabla u|}{u}\right)^{n-1} d \sigma,
$$

which is the isoperimetric inequality $(\star)$ with respect to the metric $\tilde{g}$ defined by (3).

REMARS. We have proved that with respect to $\tilde{g}$ the inequality $(\star)$ is fulfilled for every regular domain, not just for domains with volumes greater than a fixed positive constant. However, we cannot guarantee that $\tilde{g}$ is regular outside of $C$ and that it is nondegenerate. The metric loses these properties at critical points of $u$. But (as we are informed by experts) the set of critical points of $n$-harmonic functions has yet to be described when $n>2$.

To overcome this difficulty, we introduce a small positive perturbation to $\tilde{g}$ and obtain a regular nondegenerate metric with only an arbitrary small positive change of volume of the manifold. 
Then, using $(\star)$, already proved for the old "Inetric", we obtain the inequality

$$
\tilde{V}(D)<\tilde{S}(\partial D)+\varepsilon
$$

fulfilled for every regular domain $D$ with respect to the new regular nondegenerate metric.

One can now easily pass from a metric satisfying $(\star \star)$ to one where $(\star)$ holds for regular domains with volumes greater than a fixed positive number (which can be arbitrary small).

Let $m$ be a positive integer. If $\dot{V}(D)>m \varepsilon$, then by $(\star \star)$ we have an inequality $a_{m} \tilde{V}(D)<\tilde{S}(\partial D)$, where $a_{m}=(m-1) / m$. Multiplying the metric by $a_{m}^{2}$, which can be chosen arbitrary close to one, we obtain a regular nondegenerate metric conformally equivalent to the initial metric which satisfies an isoperimetric inequality of the form $(\star)$. The inequality holds for regular domains with volume greater than $m \varepsilon$. It is clear from the construction that this positive constant can be choosen as small as we wish.

Proof of Supplement 1. Consider the set of domains $\left\{D_{t}\right\}, 0<t<$ 1 , where $D_{t}=\{x \in M \mid u>t\}$. The domains $\left\{D_{t}\right\}$ exhaust the manifold $M$ as $t \rightarrow 0$. Because

$$
\tilde{S}\left(\partial D_{t}\right)=-\int_{\partial D_{t}}|\nabla u|^{n-2} \frac{\langle\nabla u, \vec{\nu}\rangle}{u^{n-1}} d \sigma
$$

the formula (4) applied to the set $U=D_{t} \backslash C$ reduces to

$$
\tilde{V}\left(D_{t}\right)=\tilde{S}\left(\partial D_{t}\right)-\tilde{S}(C) \text {. }
$$

Since $\tilde{S}\left(\partial D_{t}\right)=1 / t^{n-1}$, where

$$
I=-\int_{\partial D_{t}}|\nabla u|^{n-2}\langle\nabla u, \bar{\nu}\rangle d \sigma
$$

in view of (2), the integral $I$ (the flow) does not depend on $t$. Therefore, $\tilde{S}\left(\partial D_{t}\right) \rightarrow \infty$ as $t \rightarrow 0$. Thus, (5) implies that the manifold $(M, \tilde{g})$ has infinite volume and the isoperimetric inequality $(\star)$ is asymptotically sharp.

Note that $\partial D_{t}$ can be irregular only at critical points of $u$. By one of the known generalizations of Sard's theorem (see [10]), we may assert that for almost all $t \in(0,1)$ the set of critical points of $u \in C^{1}$ lying on $\partial D_{t}$, is a set of $(n-1)$-Hausdorff measure zero. Therefore, it is possible to vary 
the boundary $\partial D_{t}$ near these points and obtain a domain with the piecewise smooth boundary, and with almost the same values of $\dot{V}\left(D_{t}\right)$ and $\dot{b}\left(\partial D_{t}\right)$. In view of $(5)$, the asymptotic version of the isoperimetric inequality $(\star)$ will hold on this set of domains with regular boundaries.

REMARK. We have proved Supplement 1 for a metric $g$ that is not necessarily completely regular. By selecting from $\left\{D_{t}\right\}$ a sequence of domains exhausting $M$, e.g., $\left\{D_{k^{-1}}, k=1,2 \ldots\right\}$, and following the regularization process described in the previous remark, one can perturb the metric $\tilde{g}$ and obtain a regular metric with an arbitrary small change of $\tilde{S}\left(\partial D_{k^{-1}}\right)$ and $\tilde{V}\left(D_{k-1}\right)$. Thus, by (5), the ratio of these two quantities, with respect to the new metric, tends to 1 as $k \rightarrow \infty$.

It remains to note the following. After the regularization process we obtain, with respect to the new regular metric, the inequality $(\star \star)$ but not $(\star)$. But, as indicated in previous remark, one can multiply the metric by a number arbitrary close to one, and obtain a metric satisfying $(\star)$.

The ratio $\tilde{S}\left(\partial D_{k^{-1}}\right) / \tilde{V}\left(D_{k^{-1}}\right)$ is almost constant, and by (5) it can be as close to 1 as one wishes. Thus, $(\star)$ is asymptoticaly sharp with respect to the regular metric constructed above (see Supplement 1).

Proof of Supplement 2. Taking into account that in the process of regularisation of the metric $\tilde{g}$ the volume of the manifold changes as little as we wish, it is sufficient to prove Supplement 2 only for the metric $\tilde{g}$ defined in $(3)$.

Considering again the family $\left\{D_{t}\right\}$, we compute the rate of growth of $\tilde{V}\left(D_{t}\right)$ as $t \rightarrow 0$.

By virtue of the general co-area formula (see [10] or [11]),

$$
\tilde{V}^{\prime}\left(D_{t}\right)=-(n-1) \frac{\tilde{S}\left(\partial D_{t}\right)}{t},
$$

which together with (5) leads to the differential equation

$$
\tilde{V}^{\prime}\left(D_{t}\right)=-(n-1) \frac{\tilde{V}\left(D_{t}\right)+c}{t}
$$

where $c=\tilde{S}(C)$. Integrating this we find that

$$
\ln \frac{\tilde{V}\left(D_{t}\right)+c}{c}=(n-1)|\ln t| .
$$


Using the definition of $\tilde{g}$, one can easily check that the right hand side of this last equality coincides with $\dot{g}$-distance $r=r(t)$ of the set $\partial D_{t}$ from $C$. Therefore, we have

$$
\tilde{V}\left(D_{t}\right) \sim c \cdot c^{r} \text {, as } t \rightarrow 0
$$

But for the $\tilde{g}$-geodesic ball $B(r)$ of radius $r$ centered at a point of the set $C$, the following inclusions hold

$$
B(r) \subset D_{t(r)} \subset B(r+d),
$$

where the constant $d$ is the $\tilde{g}$-diameter of $C$. In view of the asymptotics of $\tilde{V}\left(D_{t}\right)$ obtained above, we conchide that

$$
c \cdot e^{r-d} \leq \tilde{V}(B(r)) \leq c \cdot e^{r}
$$

for $r$ large enough, i.e., $\tilde{V}(B(r)) \asymp e^{r}$ as $r \rightarrow \infty$.

Proof of Corollary. First, notice that in virtue of our Theorem, the conformal isoperimetric dimension of the conformally hyperbolic manifold equals $+\infty$.

The converse follows easily from the estimate (1) for the conformal capacity. In fact, if the manifold $M$ (of infinite volume) possesses an isoperimetric function of the form $P(x)=x^{\frac{m-1}{m}}, x>\varepsilon$, with $m>n$, then the integral on the left of (1) converges as $r \rightarrow+\infty$. Therefore, the manifold $M$ is of conformally hyperbolic type.

4. Concluding remarks. The previous remarks already touched on the problem of possible singularities of $\tilde{g}$ and we discussed one possible method of regularization.

Notice that the metric $\tilde{g}$ can be incomplete. However the metric completion of the space $(M, \tilde{g})$ adds to $M$ a small set of casual parabolic boundary elements (of point-type) and converts $M$ into a completely hyperbolic noncompact space $\bar{M}$ with a complete metric $\bar{g}$ on it. The isoperimetric inequality ( $\star$ ) will also hold on the completed manifold $(\bar{M}, \bar{g})$ for every domain $D$ of finite volume and finite area boundary.

In particular, this implies that our Theorem remains valid even if one removes compactness condition from the definition of regular domain.

Finally notice that if the manifold $(M, g)$ is not completely hyperbolic then there does not exist a complete Riemannian metric on $M$ conformally 
equivalent to the initial metric of the manifold and satisfying the isoperimetric property $(\star)$.

We shall discuss these topics and related problems in a paper devoted to a more complete account of our results.

Acknowledgments. The second author wishes to thank the Institut des Hautes Études Scientificues (IHES, France) for the hospitality while this manuscript was written, and I. Vardi who corrected the initial English text.

This work is also supported in part by Russian Basic Research Foundation project 99-01-01179.

\section{References}

[1] V. M. Kesel'man, V. A. Zorich: On the conformal type of Riemannian manifold, (Russian), Funk. Anal. i Prilozh., 30, (1996), no. 2, 40-55. English translation: Funct. Anal. Appl., 30, (1996), no. 2, 106-117.

[2] V. A. Zorich: Asymptotic geometry and conformal types of CarnotCarathéodory spaces, to appear in GAFA, Geom. funct. anal. 9, (1999).

[3] R. Grimaldi, P. Pansu: Sur la croissance du volume dans une classe conforme, J. Math. Pures Appl. (9) 71, (1992), no 1, 1-19.

[4] M. Gromov: Structures métriques pour les variétés riemanniennes, Notes du cours rédigé par J.Lafontaine et P.Pansu, CEDIC/Fernand-Nathan et Soc. Math. de France, Paris, 1981.

[5] V. M. Kesel'man, V. A. Zorich: Conformal type and isoperimetric dimension of a Riemannian manifold, (Russian), Mat. Zametki, 63, (1998), no. 3, 379-385. English translation: Math. Notes, 63, (1998), no. 3-4, 333337.

[6] V.G. Maz'ya: Sobolev spaces, Springer-Verlag, Berlin-HeidelbergTokyo, 1985.

[7] Yu.G. Reshetnyak: Space Mappings with Bounded Distortion, Translations of Mathematical Monographs 73, Amer.Math.Soc.; Providence, R.I., 1989.

[8] O.A. Ladyzhenskaya, N.N. Ural'tseva: Linear and quasilinear elliptic equations, Academic Press, New York-London, 1968.

[9] J. Heinonen, T. Kilpelainen, O. Martio: Nonlinear potential theory of degenerate elliptic equations, Oxford University Press, 1993. 
[10] Yu.D. Burago, V.A. Zalgaller: Ceometric inequalitics, SpringerVerlag, Berlin-New York, 1988.

[11] H. Federer: Ceometric measure theory, Springer-Verlag New York Inc., New York, 1969.

Vladimir M. Kesel'man

Department of Mathematics Kemerovo State University 650043 Kemerovo

Russia
Vladimir $\Lambda$. Zorich

Department of Mechanics and Mathematics Moscow State University

119899 Moscow

Russia 